\title{
Optimising offsets and bandwidths in vehicle traffic networks
}

\author{
Robert Burdett $^{1} \quad$ Bradley Casey $^{2} \quad$ Kai Helge Becker ${ }^{3}$
}

(Received 29 July 2014; revised 10 December 2014)

\begin{abstract}
Bandwidths and offsets are important components in vehicle traffic control strategies. This article proposes new methods for quantifying and selecting them. Bandwidth is the amount of green time available for vehicles to travel through adjacent intersections without the requirement to stop at the second traffic light. The offset is the difference between the starting-time of "green" periods at two adjacent intersections, along a given route. The core ideas in this article were developed during the 2013 Maths and Industry Study Group in Brisbane, Australia. Analytical expressions for computing bandwidth, as a function of offset, are developed. An optimisation model, for selecting offsets across an arterial, is proposed. Arterial roads were focussed upon, as bandwidth and offset have a greater impact on these types of road as opposed to a full traffic network. A generic optimisation-simulation approach is also
\end{abstract}

http://journal . austms.org.au/ojs/index.php/ANZIAMJ/article/view/8917 gives this article, (c) Austral. Mathematical Soc. 2014. Published December 16, 2014, as part of the Proceedings of the 2013 Mathematics and Statistics in Industry Study Group. ISSN 1446-8735. (Print two pages per sheet of paper.) Copies of this article must not be made otherwise available on the internet; instead link directly to this URL for this article. 
proposed to refine an initial starting solution, according to a specified metric. A metric that reflects the number of stops, and the distance between stops, is proposed to explicitly reduce the dissatisfaction of road users, and to implicitly reduce fuel consumption and emissions. Conceptually the optimisation-simulation approach is superior as it handles real-life complexities and is a global optimisation approach. The models and equations in this article can be used in road planning and traffic control.

\section{Contents}

1 Introduction

M78

2 Research trends

M81

3 Bandwidths

M87

3.1 Introduction . . . . . . . . . . . . . . . M87

3.2 Quantification of bandwidth and pair-wise analysis . . . M89

3.3 Beyond pair-wise analysis . . . . . . . . . . . M95

4 A general optimisation and refinement approach

M97

5 Conclusions

M102

References

M105

\section{Introduction}

Traffic signalisation is a regulated mechanism to control the movement of large numbers of vehicles on road and rail networks. Traffic signals are positioned at road or rail intersections to stop collisions (i.e., conflicting traffic movements) from occurring on intersecting paths; otherwise they are used to separate and 
improve (i.e., smooth) the flow of vehicles. Pfeifeng et al. [16] stated that the idea of coordinating traffic signals is to keep traffic progressing in a platoon, minimizing delays or stops throughout the signal system. They similarly report that "Coordinated signal systems operate most efficiently when traffic volumes between adjacent intersections are heavy and signalized intersections are in proximity to each other". Traffic detectors are used to measure flows but they do not measure demand [10].

There are two main approaches for controlling traffic with signals, namely reactive and proactive. Reactive approaches change signals timings as a consequence of changes in traffic conditions. However, a proactive approach predicts what is likely to occur and changes the timings beforehand. In the context of roads, signal control strategies are manifested as cycle times (denoted as $c_{i}$ ), phase splits (denoted as $p_{i, j}$ ), and offsets (denoted as $o_{i, j, i^{\prime}, j^{\prime}}$ ), where the indices $i$ and $i^{\prime}$ refer to intersections, while the indices $j$ and $j^{\prime}$ refer to particular phases at intersections $i$ and $i^{\prime}$, respectively. A general offset may also be imposed at each intersection and is denoted simply as $\boldsymbol{o}_{i}$.

These control strategies are either fixed or variable (i.e., adaptive). Their selection is typically dependent on current or perceived traffic volumes and conditions (such as time of day, weather, and accidents). Each intersection has different phases of traffic flow. Each phase is associated with the movement of traffic through the intersection from a specific direction (i.e., a green period). The time to perform "green" periods for all the phases at an intersection is the cycle time. Each phase has a cycle of red, green and yellow lighting time that is repeated. The time for each of these periods is not necessarily the same; this is dictated by the phase split. The magnitude of the splits dictates the flow of traffic in specific directions. In addition there may be a different number and ordering of phases at each intersection. Offsets are a measure of the "time" difference of the beginning of successive "green" periods between adjacent intersection phases and along a given (common) route. In other words two phases have a common offset if traffic passes through each intersection in the same direction. Figure 1 illustrates this information. 


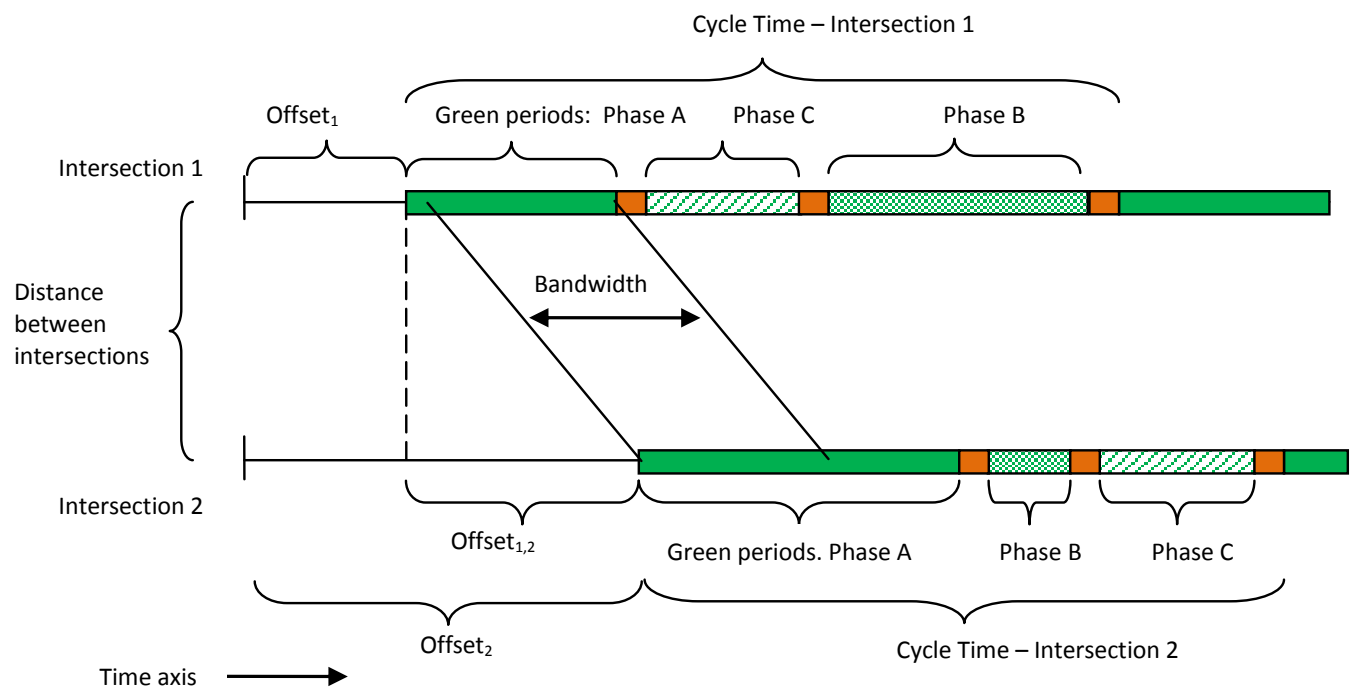

Figure 1: A diagram describing cycles, offsets, phases and bandwidths.

The choice of signalisation parameters is difficult because road networks are time-varying and stochastic. In other words, the volume of traffic is constantly changing throughout each and every day and is not easily predicted. Optimisation of these parameters is also difficult because the decision problem is not well defined, is not deterministic, and is computationally intractable. Furthermore there are a variety of system performance metrics (i.e., key performance indicators); these are not easily computed and the best one is arguable and situation/client dependent.

Simulation is the dominant and primary tool for evaluating alternative signal timings (i.e., solutions). Macroscopic simulators are based on fluid dynamics, since they consider traffic flow as a continuous fluid. The second paradigm is the one that includes microscopic simulators. For them, traffic is considered as a collection of discrete particles following some rules about their interaction. Sanchez et al. [18] reported that macroscopic simulators cannot model the discrete dynamics that arises from the interaction among individual vehicles. 
Section 2 provides a review of the literature on traffic control and optimisation. In Section 3 we propose an analytical approach for quantifying bandwidths and develop alternative metrics for measuring signal timing efficiency and approximating driver dissatisfaction. Section 4 presents a generic optimisation approach as a means of optimising offsets.

\section{Research trends}

Table 1 summarises recent research in this field. This is not a comprehensive list of all work that has been done. However, it is indicative of current trends and directions. The dashes ("-") signify undefined, unknown or irrelevant information. A description of the headings in this table is as follows.

SYS. $\quad$ System, computerised platform, GUI

ALG. $\quad$ Algorithmic approach, i.e., customised approach

SIM. Simulation model from one of DES, petri, statistical, cell transmission model (CTM), cellular automata (CA), other

OPT STR. Optimisation strategy. Includes meta-heuristic global optimisation approaches such as evolutionary algorithm (EA), genetic algorithm (GA), simulated annealing (SA), tabu search (TS), hybrid and constructive heuristics $(\mathrm{CH})$. Also includes mathematical programming techniques such as mixed integer linear (MILP).

CTRL STR. Proactive (predictive), reactive, analytical, off-line, on-line, static (i.e., deterministic), graph theoretic, etc.

The articles in Table 1 are now discussed.

Wey [22] developed an approach for network traffic signal optimisation. The problem was formulated as a mixed integer model. As the model has the form of a linear multi-commodity network flow problem, a modified network simplex algorithm and a branch and bound approach were used to solve the model. 


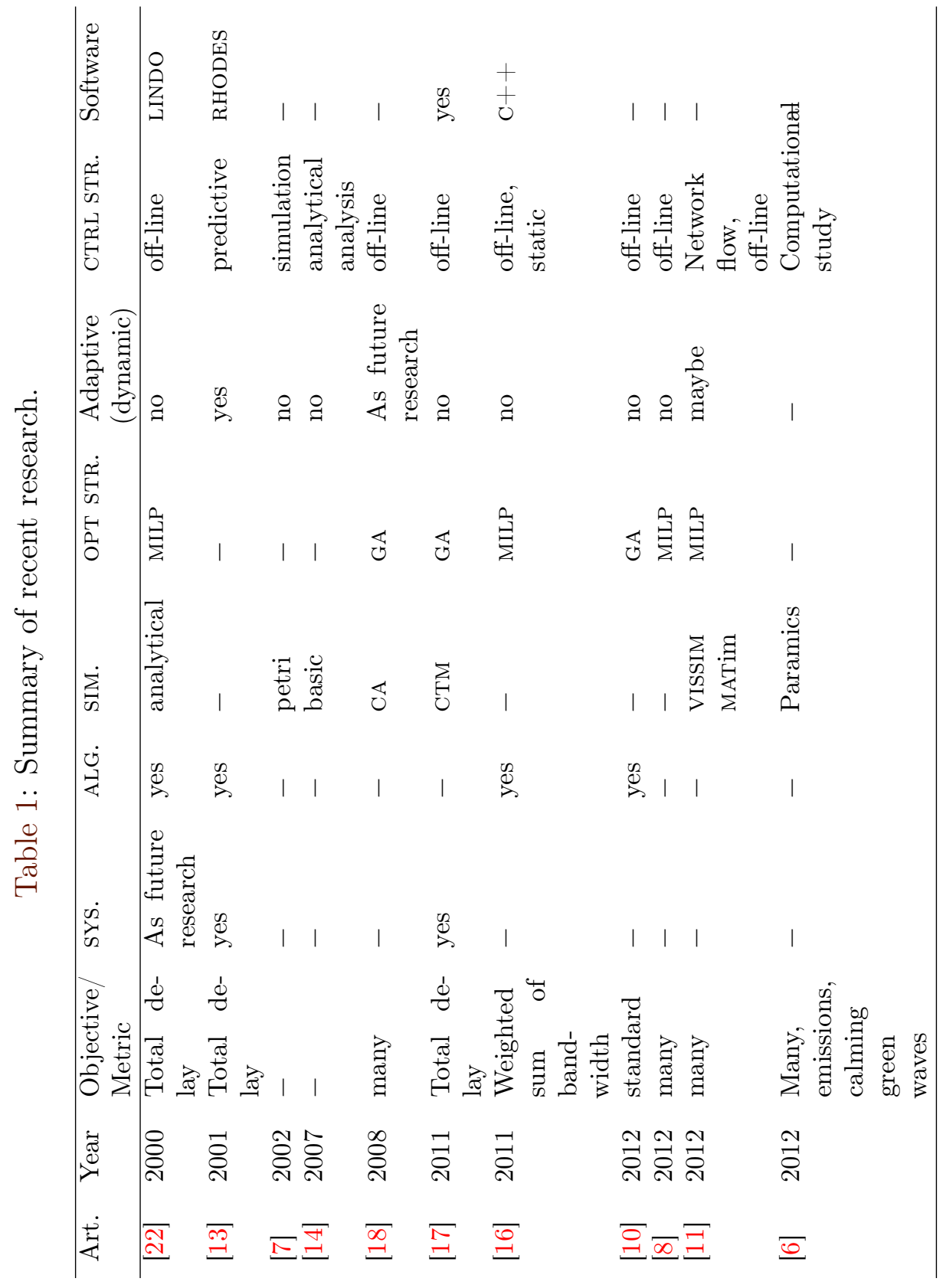


Mirchandani and Head [13] reported details of their real-time traffic-adaptive signal control system, called RHODES (development over six years). This system has a variety of components. One component named PREDICT is used to predict future arrivals at intersections. It utilises detectors upstream of intersections and the current signal timings. A dynamic programming algorithm (COP) is used at the lowest level of RHODES for signal-timing optimisation at intersections (i.e., intersection control logic). A component called REALBAND optimises the movement of observed platoons in the subnetwork and attempts to form progression bands. These decisions are input as constraints in COP. A simulation testing procedure/model called CORSIM was used to evaluate RHODES.

Di Febbraro et al. [7] developed timed petri net models for the simulation of urban traffic systems regulated by signalised intersections. Elementary structural components were developed for intersections and road stretches and these can be replicated to create a full traffic network. Offsets can be embedded within the structure of the petri net model. However, no implementation details were provided and there was no realistic (full scale) case study and application of the proposed simulation strategy.

Nagatani [14] investigated vehicle movements through an infinite set of green wave lights on a single lane roadway. Cycle times and offsets were altered and the results (i.e., patterns) were analysed. Some analytical expressions were developed to describe arrival times, tour times, etc.

Sanchez et al. [18] considered the optimisation of traffic light cycles and proposed a Genetic algorithm (GA) technique with a micro simulator. The article provides a good description of the state of the art in the early 2000s, and those articles published at that time. The GA approach uses a chromosome solution representation (binary gray encoding) and is quite basic. For instance it uses standard crossover operators, mutation and algorithm parameter control. The article provides two minor case studies.

Rohde and Friedrich [17] applied a cell transmission model (СTM) and an offset optimisation approach (GA). These were embedded in a GUI and 
were shown to be successful. The software includes four main components: Net-Editor, Traffic Signal-Editor, CTM-Control and Offset Optimizer and Output Visualization. The CTM determines queuing like statistics. The overall delay in the network was the chosen objective. The GA approach was quite conventional and did not provide any innovation. The proposed optimisation approach was applied to a German arterial road case study. The objective of the offset optimization was to improve northbound traffic flow towards the city centre in the morning peak hour. The optimized offsets and fixed time control programs provided a reduction in travel times in the test area of $19 \%$, whereas AIMSUN (see the list of software) estimated $28 \%$. The difference was attributed to difficulties in optimizing offsets between fixed time and actuated traffic controlled intersections. Hence further research was reported as necessary to model traffic actuated control with the CTM.

Peifeng et al. [16] developed an arterial coordination model and a network coordination model to obtain the best signal timing solutions for traffic handling on arterials and street networks. The main objective of the approaches was to provide the maximum bandwidth progression by adjusting the offset and phase sequence patterns for each signalized intersection. The model used average/static values for parameters (i.e., volumes and capacities) and did not address variability. The solution of the models (i.e., solution approach and software) was not commented upon and the numerical investigations were quite minimal and inconsequential. Simulation was not utilised in this approach.

Johnston and Suter [10] provided a thorough explanation of road control and signalisation issues. They applied a Genetic algorithm and clustering techniques. A Brisbane road was analysed in their case study. Microsoft Excel was heavily utilised for calculations. The software Transyt- $7 \mathrm{f}$ was also used for verification. Implementation and other technical details of the solution approach were not explained in this article.

Gentili and Mirchandani [8] considered where (and how many) sensors should be placed in order to observe and estimate accurately the traffic flows on road 
networks. Ten mathematical models were considered and/or developed and a number of theorems were proposed. Open research and future directions were commented upon. A problem categorisation was also made.

Kohler and Strehler [11] consider the application and combination of static and dynamic models for traffic control and optimisation. A cyclically timeexpanded network was proposed as a traffic flow model that simultaneously optimises traffic assignment and signal coordination with exact mathematical programming techniques. VISSIM and MATSim simulation software was used for validation.

Coensel et al. [6] considered the effects of synchronized traffic lights on emissions and performed a computational study. The traffic intensity and green split were found to have the largest influence on emissions, while the cycle time did not have a significant influence on emissions. Coensel et al. provided significant details about recent research and is quite comprehensive.

Traffic control and planning software are summarised below.

\section{TRANSYT (Traffic Network Study Tool)}

Used for designing, modelling and coordination of traffic signals, ranging from individual isolated junctions to large complex networks. It can quickly asses individual junction performance and produces optimum fixed-time co-ordinated traffic signal timings in any network of roads for which traffic flows are known. Off-line. http://www.trl.co.uk/ Transyt.htm

\section{AIMSUN}

An integrated transport modelling software. It performs traffic assignment at a macroscopic level, mesoscopic and microscopic simulation, dynamic traffic assignment, as well as other features. It produces outputs as images, videos, tables or raw data. http://www.aimsun.com/wp/

\section{SCOOT (Split, Cycle, and Offset Optimization Technique)}

An adaptive traffic control system. Developed by the Transport Research Lab (TRL). Software minimises stops and delays. Uses similar model to 
TRANSYT, that is, uses output from model as input into signal timing optimisers. Optimisers make a series of small adjustments to signal timings to minimise modelled vehicle delays throughout the network. http://www.scoot-utc.com/

\section{PASSER}

Designed by Texas Transportation Institute for traffic control optimization. There have been four versions to date.

\section{MAXBAND}

Developed by Little, Kelson \& Gartner, 1981

\section{SCATS (Sydney Coordinated Adaptive Traffic System)}

Developed and maintained by Nsw Department of Main Roads, now Roads and Maritime Services. http://www.scats.com.au/

\section{STREAMS (Integrated Intelligent Transport System)}

Developed by the Department of Main Roads Queensland. Privatised in 2002 to form Transmax. This enterprise traffic management system is designed to monitor, control and manage traffic on streets and highways. It performs adaptive traffic management and chooses the optimal traffic plan from a selection of plans created by users. http://www.transmax. com.au/

Our investigation of recent papers revealed the following.

1. Genetic algorithms are very popular. Other meta-heuristics seem not to have been applied. Evolutionary approaches [3, 4], which are superior to GA, have not been applied either.

2. There are few full scale systems developed from academic research; RHODES is the exception.

3. Almost all the approaches are not adaptable to real time traffic conditions.

4. The choice of simulation tool and performance metric is variable, that 
is, matter of personal preference and not scientific reasoning.

5. Analytical approaches feature quite regularly but simulation (coupled with an optimisation strategy) is most prominent.

\section{Bandwidths}

\subsection{Introduction}

Bandwidth [16] is commonly defined as the amount of green time available for vehicles to travel between two intersections at a specified speed. It is useful to quantify these bandwidths for every pair of adjacent intersections. A differentiation between bandwidth in each direction is also necessary. We carry out an analysis that performs separate "pair-wise" calculations in this section.

Let $g_{i, j}$ be the duration of the green phase for phase $j$ at intersection $i$. Letting $p_{i, j}$ be the percentage split, then $g_{i, j}=c_{i} \times p_{i, j}$. Let $s_{i, j}$ be the time that the first green phase starts for phase $j$ at intersection $i$, relative to the offset $o_{i}$ of the intersections cycle. Similarly denote et ${ }_{i, j}$ as the time that the first green phase ends for phase $j$ at intersection $i$, relative to the offset $o_{i}$ of the intersection cycle. Therefore,

$$
\begin{aligned}
\text { et }_{i, j} & =s t_{i, j}+g_{i, j}, \\
s t_{i, j} & =\sum_{j^{\prime}=1}^{j-1}\left(g_{i, j^{\prime}}+a_{i, j^{\prime}}+\mu_{i, j^{\prime}}\right) .
\end{aligned}
$$

In equation (2) $a_{i, j}$ is the duration of the amber phase, and $\mu_{i, j}$ is the duration of a safety buffer, separating phases, whereby no traffic is moving. In this article we assume that phase one occurs first, then phase two, and so on. For the $n$th occurrence of phase $\mathfrak{j}$;

$$
\mathrm{st}_{i, j}^{n}=\mathrm{st}_{i, j}+(\mathrm{n}-1) \mathrm{c}_{\mathrm{i}} \text { for } \mathrm{n}=1,2, \ldots,
$$



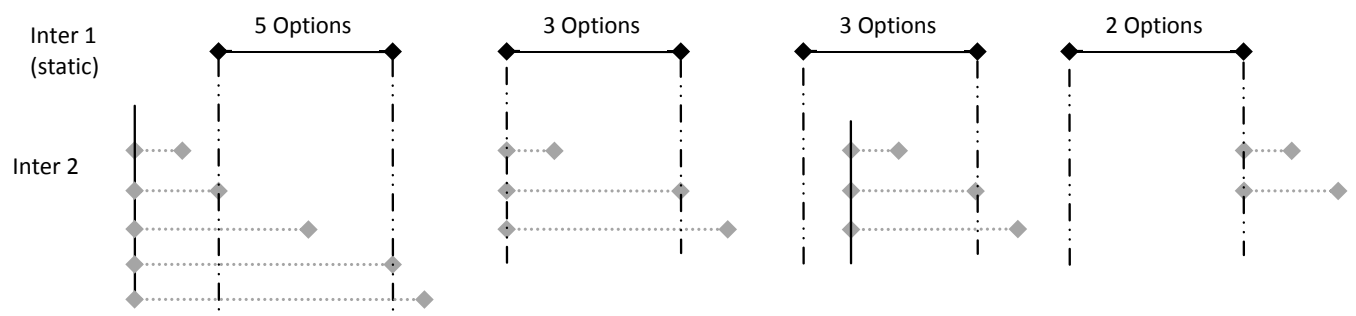

Figure 2: The comparative position of green periods at two adjacent intersections.

$$
\mathrm{et}_{i, j}^{n}=\mathrm{st}_{i, j}^{n}-\mathrm{g}_{i, j} \text { for } \mathrm{n}=1,2, \ldots
$$

Bandwidth occurs between green phases at adjacent intersections. This is then a comparison of two general time intervals, each starting and ending at computable times. Figure 2 illustrates (for a fixed green period at intersection 1) all the possible scenarios for which bandwidths may be quantified. The $x$-axis is referring to durations of time. Depending on the travel time between the intersections, different bandwidth times are obtained, and may even be zero. Table 2 exactly quantifies the thirteen cases. However, upon closer scrutiny, cases which result in a non-zero bandwidth are quantified more simply by the four universal cases shown in Figure 3.

The four reduced cases are as follows.

Case A: Bandwidth determined by start time at intersection 1 and end time at intersection 1 , that is, band $=e_{i, j}-s_{i, j}$. Condition: $s_{i^{\prime}, j^{\prime}} \leqslant$ st $_{i, j}+\theta_{i, i^{\prime}}$ and et $_{i^{\prime}, j^{\prime}} \geqslant$ et $_{i, j}+\theta_{i, i^{\prime}}$.

Case B: Bandwidth determined by start time at intersection 1 and end time at intersection 2 , that is, band $=$ et $_{i^{\prime}, j^{\prime}}-s_{i, j}-\theta_{i, i^{\prime}}$. Condition: $s_{i^{\prime}, j^{\prime}} \leqslant s t_{i, j}+\theta_{i, i^{\prime}}$ and $e_{i^{\prime}, j^{\prime}}<$ et $_{i, j}+\theta_{i, i^{\prime}}$.

Case C: Bandwidth determined by start time at intersection 2 and end time at intersection 1 , that is, band $=\mathrm{et}_{i, j}-\mathrm{st}_{\mathrm{i}^{\prime}, j^{\prime}}+\theta_{i, i^{\prime}}$. Condition: $s t_{i^{\prime}, j^{\prime}}>s_{i, j}+\theta_{i, i^{\prime}}$ and $e_{i^{\prime}, j^{\prime}}>$ et $_{i, j}+\theta_{i, i^{\prime}}$. 


\section{Case A}

Inter 1

Inter 2

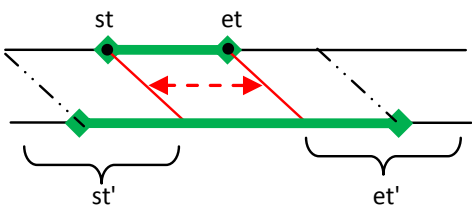

Case B

Inter 1

Inter 2

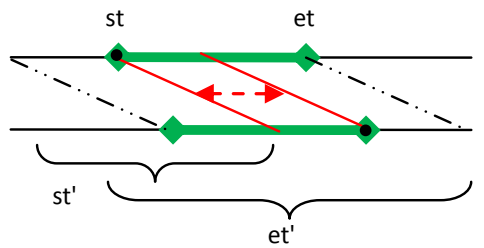

Case C

Inter 1

Inter 2

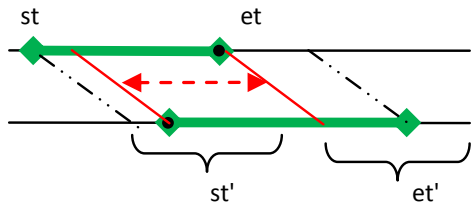

Case D

Inter 1

Inter 2

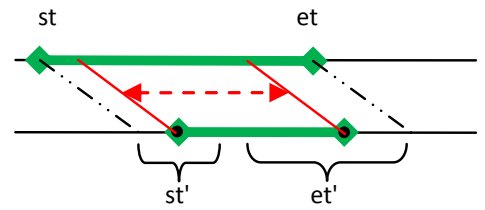

Figure 3: Reduced cases for determining non zero bandwidths.

Case D: Bandwidth determined by start time at intersection 2 and end time at intersection 2, that is, band $=$ et $_{i^{\prime}, j^{\prime}}-s_{i^{\prime}, j^{\prime}}$. Condition: $s_{i^{\prime}, j^{\prime}}>$ st $_{i, j}+\theta_{i, i^{\prime}}$ and et $_{i^{\prime}, j^{\prime}} \leqslant$ et $_{i, j}+\theta_{i, i^{\prime}}$.

The travel time between intersections is denoted by $\theta_{i, i^{\prime}}$. In Figure 3 , et ${ }^{\prime}=$ et $_{i^{\prime}, j^{\prime}}$ and $s t^{\prime}=s t_{i^{\prime}, j^{\prime}}$.

\subsection{Quantification of bandwidth and pair-wise analysis}

An expression is derived for the bandwidth band ${ }_{i, j, i^{\prime}, j^{\prime}}$ between the green phases $\boldsymbol{j}$ and $\boldsymbol{j}^{\prime}$ of intersections $\boldsymbol{i}$ and $\boldsymbol{i}^{\prime}$, respectively. We assume that both intersections have the same cycle time $c$. Otherwise the bandwidth would not be constant over time in the general case because the starting points of the green phases of $\boldsymbol{j}$ and $\boldsymbol{j}^{\prime}$ of intersections $\boldsymbol{i}$ and $\boldsymbol{i}^{\prime}$ would permanently shift against each other. In other words, the bands given in Figure 3 would not necessarily be the only bands of uninterrupted traffic flow through the two intersections $i$ and $i^{\prime}$. However, under the assumption of a common cycle time $\mathrm{c}$, the bandwidth is a well defined function of 
- the offsets $o_{i, j, i^{\prime}, j^{\prime}}=s t_{i^{\prime}, j^{\prime}}-s t_{i, j}$ of the intersections,

- the travel time $\theta_{i, i^{\prime}}$ between the intersections,

- the lengths $g_{i, j}$ and $g_{i^{\prime}, j^{\prime}}$ of the green phases of the intersections, and

- the common cycle time c.

Trivially, the common cycle time $c$ is an upper bound on the bandwidth function, that is,

$$
\operatorname{band}_{i, j, i^{\prime}, j^{\prime}}\left(g_{i, j}, g_{i^{\prime}, j^{\prime}}, c, \theta_{i, i^{\prime}}, o_{i, j, i^{\prime}, j^{\prime}}\right) \leqslant c,
$$

and the bandwidth function attains the value $c$ if and only if $g_{i, j}=g_{i^{\prime}, j^{\prime}}=c$. The only other case where the bandwidth is constant that does not depend on the offset is the case where $\max \left(g_{i, j}, g_{i^{\prime}, j^{\prime}}\right)=c$, but $\min \left(g_{i, j}, g_{i^{\prime}, j^{\prime}}\right)<c$, and here we have

$$
\operatorname{band}_{i, j, i^{\prime}, j^{\prime}}\left(g_{i, j}, g_{i^{\prime}, j^{\prime}}, c, \theta_{i, i^{\prime}}, o_{i, j, i^{\prime}, j^{\prime}}\right)=\min \left(g_{i, j}, g_{i^{\prime}, j^{\prime}}\right) \text {. }
$$

It remains to discuss the case $g_{i, j}, g_{i^{\prime}, j^{\prime}}<c$, that is, the case where the bandwidth is a (non-constant) function of the offset. Let us start, by considering the situation where both intersections share the same location, where, unrealistically, the travel time between the intersections is $\theta_{i, i^{\prime}}=0$, and, additionally, where the length of the green phase of intersection $i$ is greater or equal the length of the green phase at intersection $i^{\prime}$, that is, $g_{i, j} \geqslant g_{i^{\prime}, j^{\prime}}$. If there is no offset between the intersections, that is, $o_{i, j, i^{\prime}, j^{\prime}}=0$, then we have the situation of Figure 4a, where the band is indicated as a shaded area and the bandwidth is equal to $g_{i^{\prime}, j^{\prime}}$. Introducing an offset $o_{i, j, i^{\prime}, j^{\prime}}>0$ leads to the situation in Figure 4b. As long as the offset is small enough such that et $t_{i^{\prime}, j^{\prime}}$ does not exceed et $t_{i, j}$, the bandwidth remains on the level $g_{i^{\prime}, j^{\prime}}$.

When $o_{i, j, i^{\prime}, j^{\prime}}$ increases beyond the point where $e_{i^{\prime}, j^{\prime}}=e_{i, j}$ (as in Figure $4 b$ ), the bandwidth decreases by one minute for each minute that the offset $\Delta$ increases. With increasing $o_{i, j, i^{\prime}, j^{\prime}}$, this process continues either until the green phases of the two intersections no longer overlap and the bandwidth has 
reached a minimum of $b_{\min }=0$ (Figure 4c), provided that $g_{i, j}+g_{i^{\prime}, j^{\prime}}>\mathbf{c}$. When et $_{i^{\prime}, j^{\prime}}=c$, the green phase of intersection $i^{\prime}$ begins to overlap with the green phase of intersection $i$ that belongs to the next cycle (Figure $4 \mathrm{~d}$ ). In this latter case the bandwidth reaches a minimum of $b_{\min }=g_{i, j}+g_{i^{\prime}, j^{\prime}}-c$.

In both cases once the minimum is attained, we can increase the offset $o_{i, j, i^{\prime}, j^{\prime}}$ further by $\left|g_{i, j}+g_{i^{\prime}, j^{\prime}}-c\right|$ units of time without any change in the bandwidth. (In the former case this period of constant minimal bandwidth lasts from st $_{i^{\prime}, j^{\prime}}=$ et $_{i, j}$ until et $t_{i^{\prime}, j^{\prime}}=\mathrm{c}$, while in the latter case this period lasts from $\mathrm{et}_{i^{\prime}, j^{\prime}}=\mathrm{c}$ until $\mathrm{st}_{\mathrm{i}^{\prime}, j^{\prime}}=\mathrm{et}_{\mathrm{i}, \mathrm{j}}$.)

Beyond these points, the bandwidth starts to increase at a rate of one minute per each minute that the offset $o_{i, j, i^{\prime}, j^{\prime}}$ increases, until $o_{i, j, i^{\prime}, j^{\prime}}=c$, when the maximum bandwidth is attained as in the beginning of our analysis and the situation repeats itself with the start of a new cycle.

This setting (where we assume $\theta_{i, i^{\prime}}=0$ and $g_{i, j} \geqslant g_{i^{\prime}, j^{\prime}}$ for the moment) yields the bandwidth function depicted in Figure 4e. More precisely, it follows from our analysis that the bandwidth as a function of the offset is described by the following parameters:

$$
\begin{aligned}
& b_{\min }=\max \left(0, g_{i, j}+g_{i^{\prime}, j^{\prime}}-c\right), \quad b_{\max }=g_{i^{\prime}, j^{\prime}}, \\
& l_{\min }=\left|g_{i, j}+g_{i^{\prime}, j^{\prime}}-c\right|, \quad l_{\max }=\left|g_{i, j}-g_{i^{\prime}, j^{\prime}}\right| .
\end{aligned}
$$

So far we have considered the case $g_{i, j} \geqslant g_{i^{\prime}, j^{\prime}}$. For $g_{i, j}<g_{i^{\prime}, j^{\prime}}$ the general shape of the bandwidth function does not change. Moreover, a line of argument analogous to our analysis above that, except the maximum bandwidth, which is now $b_{\max }=g_{i, j}$, our statements about the parameters that describe the shape of the bandwidth function remain true (the other three formulas are symmetric in $g_{i, j}$ and $\left.g_{i^{\prime}, j^{\prime}}\right)$. However, the bandwidth function for $g_{i, j}<g_{i^{\prime}, j^{\prime}}$ differs from the case $g_{i, j} \geqslant g_{i^{\prime}, j^{\prime}}$ since it starts to decline immediately once we introduce an offset $o_{i, j, i^{\prime}, j^{\prime}}>0$ because the length of the interval in which the green phases of intersections $i$ and $i^{\prime}$ overlap decreases once we start moving the green phase of intersection $i^{\prime}$ to the right along the time axis. This line 


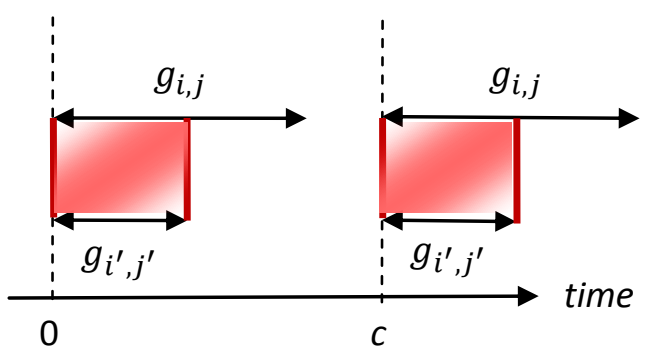

(a) $o_{i, j, i^{\prime}, j^{\prime}}=0$

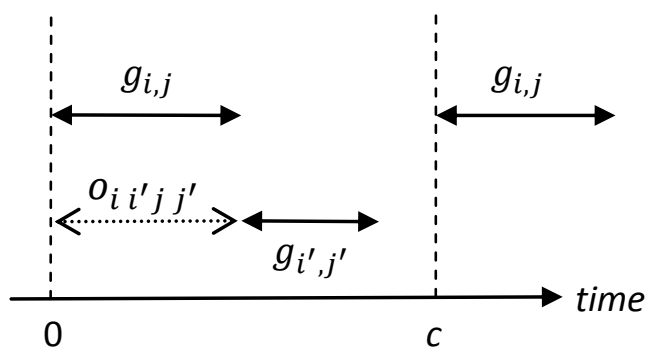

(c) $o_{i, j, i^{\prime}, j^{\prime}}>0, b_{\min }=0$

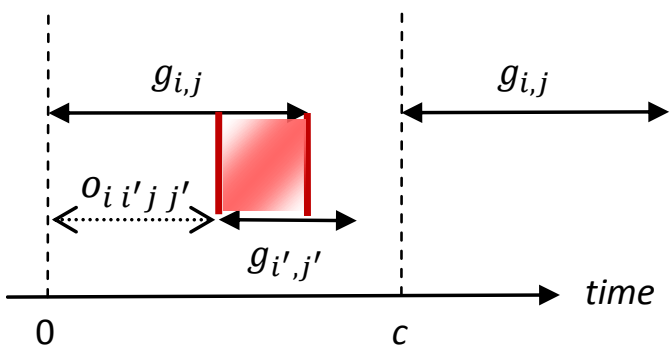

(b) $\mathrm{o}_{i, j, i^{\prime}, j^{\prime}}>0$, bandwidth decreasing

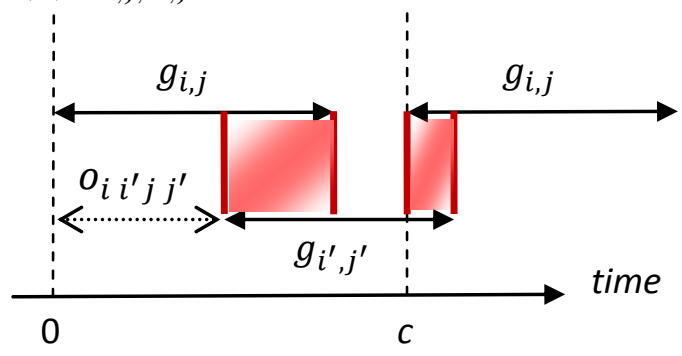

(d) $o_{i, j, i^{\prime}, j^{\prime}}>0, b_{\min }>0$

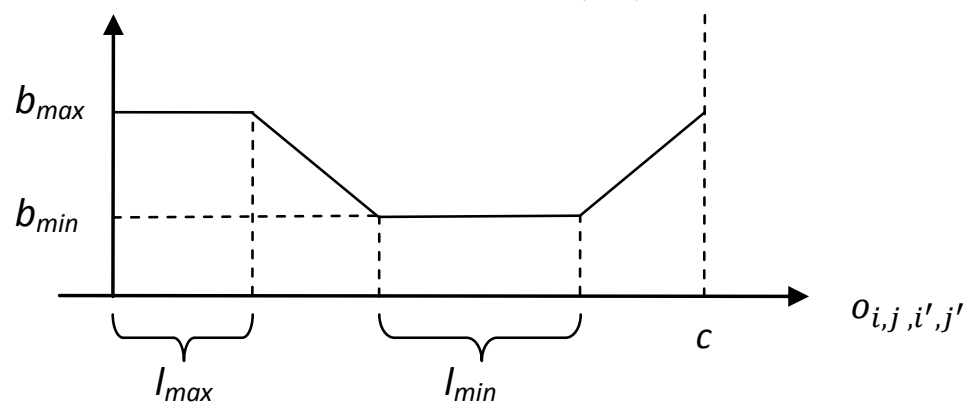

(e) $\operatorname{band}_{i, j, i^{\prime}, j^{\prime}}\left(g_{i, j}, g_{i^{\prime}, j^{\prime}}, c, \theta, o_{i, j, i^{\prime}, j^{\prime}}\right)$

Figure 4: Bandwidth as a function of offset. 
of reasoning leads to the insight that the bandwidth function for $g_{i, j}<g_{i^{\prime}, j^{\prime}}$ is obtained by shifting the function for $g_{i, j} \geqslant g_{i^{\prime}, j^{\prime}}$ by $l_{\max }$ units to the left. To summarize, the bandwidth as a function of the offset is described by the parameters

$$
\begin{aligned}
& b_{\min }=\max \left(0, g_{i, j}+g_{i^{\prime}, j^{\prime}}-c\right), \quad b_{\max }=\min \left(g_{i, j}, g_{i^{\prime}, j^{\prime}}\right), \\
& l_{\min }=\left|g_{i, j}+g_{i^{\prime}, j^{\prime}}-c\right|, \quad l_{\max }=\left|g_{i, j}-g_{i^{\prime}, j^{\prime}}\right| .
\end{aligned}
$$

Since, in the intervals where the bandwidth increases or decreases, the function has the slopes 1 and -1 , respectively, we also have,

$$
c=l_{\min }+l_{\max }+2\left(b_{\max }-b_{\min }\right) .
$$

For $g_{i, j} \geqslant g_{i^{\prime}, j^{\prime}}$ the maximum is first attained at $o_{i, j, i^{\prime}, j^{\prime}}=0$. For $g_{i, j}<$ $g_{i^{\prime}, j^{\prime}}$ the maximum is first attained at $o_{i, j, i^{\prime}, j^{\prime}}=-l_{\max }=-\left|g_{i, j}-g_{i^{\prime}, j^{\prime}}\right|$. Considering the cyclical nature of the problem, where

$$
o_{i, j, i^{\prime}, j^{\prime}}=c-l_{\max }=c-\left|g_{i, j}-g_{i^{\prime}, j^{\prime}}\right|
$$

this completes the description of the bandwidth function.

The last aspect we have to consider before an explicit expression for band $d_{i, j, i^{\prime}, j^{\prime}}$ is defined when intersections $i$ and $i^{\prime}$ are not located at the same place, that is, when it takes vehicles a time $\theta_{i, i^{\prime}}>0$ to travel from $i$ to $i^{\prime}$. This means that all vehicles arrive at the second intersection $\theta_{i, i^{\prime}}$ units of time later than in the setting considered so far and is therefore equivalent, from the perspective of a vehicle that has to pass through both intersections, to starting the green phase of the second intersection $\theta_{i, i^{\prime}}$ units of time earlier. In other words, our results above remain valid for the case $\theta_{i, i^{\prime}}>0$ if we apply the corrected offset

$$
\Delta:=\mathrm{o}_{i, j, i^{\prime}, j^{\prime}}-\theta_{i, i^{\prime}}
$$

On the basis of equations (5) to (9), an explicit description of the bandwidth function is defined. Taking into account the offset between the green phases of the intersections, the travel time between the intersections, and the additional 
shift of the bandwidth function that occurs iff $g_{i, j}<g_{i^{\prime}, j^{\prime}}$, we define the total offset

$$
\Delta^{\prime}:= \begin{cases}\Delta=o_{i, j, i^{\prime}, j^{\prime}}-\theta_{i, i^{\prime}} & \text { for } g_{i, j} \geqslant g_{i^{\prime}, j^{\prime}}, \\ \Delta+g_{i^{\prime}, j^{\prime}}-g_{i, j}=o_{i, j, i^{\prime}, j^{\prime}}-\theta_{i, i^{\prime}}+g_{i^{\prime}, j^{\prime}}-g_{i, j} & \text { for } g_{i, j}<g_{i^{\prime}, j^{\prime}}\end{cases}
$$

Due to the cyclical nature of the problem bandwidth function is mapped on itself when shifted by the common cycle time $\mathrm{c}$. This implies that it suffices to give the values of the bandwidth function only for $\Delta^{\prime} \in[0, c)$. We now state the bandwidth as a function of the (corrected) offset (cf. Figure 4e),

$$
\begin{aligned}
& \operatorname{band}_{i^{\prime}, j^{\prime}, i, j}\left(g_{i, j}, g_{i^{\prime}, j^{\prime}}, c, \theta_{i, i^{\prime}}, o_{i, j, i^{\prime}, j^{\prime}}\right)=\operatorname{band}_{i, j, i^{\prime}, j^{\prime}, g_{i, j}, g_{i^{\prime}, j^{\prime}, c}}\left(\Delta^{\prime}\right) \\
& = \begin{cases}b_{\max } & \text { for } 0 \leqslant \Delta^{\prime} \leqslant l_{\max }, \\
b_{\max }+l_{\max }-\Delta^{\prime} & \text { for } l_{\max }<\Delta^{\prime} \leqslant l_{\max }+b_{\max }-b_{\min }, \\
b_{\min } & \text { for } l_{\max }+b_{\max }-b_{\min }<\Delta^{\prime} \leqslant c-b_{\max }+b_{\min }, \\
b_{\max }-c+\Delta^{\prime} & \text { for } c-b_{\max }+b_{\min }<\Delta^{\prime} \leqslant c .\end{cases} \\
& = \begin{cases}\min \left(g_{i, j}, g_{i^{\prime}, j^{\prime}}\right) & \text { for } 0 \leqslant \Delta^{\prime} \leqslant\left|g_{i, j}-g_{i^{\prime}, j^{\prime}}\right|, \\
\min \left(g_{i, j}, g_{i^{\prime}, j^{\prime}}\right) & \text { for }\left|g_{i, j}-g_{i^{\prime}, j^{\prime}}\right|<\Delta^{\prime} \leqslant\left|g_{i, j}-g_{i^{\prime}, j^{\prime}}\right|+ \\
\left|g_{i, j}-g_{i^{\prime}, j^{\prime}}\right|-\Delta^{\prime} & \min \left(g_{i, j}, g_{i^{\prime}, j^{\prime}}\right)-\max \left(0, g_{i, j}+g_{i^{\prime}, j^{\prime}}-c\right), \\
\max \left(0, g_{i, j}+g_{i^{\prime}, j^{\prime}}-c\right) & \text { for }\left|g_{i, j}-g_{i^{\prime}, j^{\prime}}\right|+\min \left(g_{i, j}, g_{i^{\prime}, j^{\prime}}\right)- \\
& \max \left(0, g_{i, j}+g_{i^{\prime}, j^{\prime}}-c\right)<\Delta^{\prime} \leqslant \\
& c-\min \left(g_{i, j}, g_{i^{\prime}, j^{\prime}}\right)+\max \left(0, g_{i, j}+g_{i^{\prime}, j^{\prime}}-c\right), \\
\min \left(g_{i, j}, g_{i^{\prime}, j^{\prime}}\right)-c+\Delta^{\prime} & \text { for } \quad \min \left(g_{i, j}, g_{i^{\prime}, j^{\prime}}\right)+ \\
& \max \left(0, g_{i, j}+g_{i^{\prime}, j^{\prime}}-c\right)<\Delta^{\prime} \leqslant c,\end{cases}
\end{aligned}
$$

Using equation (10) and rearranging some terms yields the following expression for the bandwidth function in terms of the (original) offset $o_{i, j, i^{\prime}, j^{\prime}}$,

$$
\operatorname{band}_{i^{\prime}, j^{\prime}, i, j}\left(g_{i, j}, g_{i^{\prime}, j^{\prime}}, c, \theta_{i, i^{\prime}}, o_{i, j, i^{\prime}, j^{\prime}}\right)=\operatorname{band}_{i, j, i^{\prime}, j^{\prime}, g_{i, j}, g_{i^{\prime}, j^{\prime}}, \mathfrak{c}, \theta_{i, i^{\prime}}}\left(o_{i, j, i^{\prime}, j^{\prime}}\right)
$$




$$
= \begin{cases}\min \left(g_{i, j}, g_{i^{\prime}, j^{\prime}}\right) & \text { for } \theta_{i, i^{\prime}}+\min \left(0, g_{i, j}-g_{i^{\prime}, j^{\prime}}\right) \leqslant o_{i, j, i^{\prime}, j^{\prime}} \leqslant \\ & \theta_{i, i^{\prime}}+\max \left(0, g_{i, j}-g_{i^{\prime}, j^{\prime}}\right), \\ g_{i, j}+\theta_{i, i^{\prime}}-o_{i, j, i^{\prime}, j^{\prime}} & \text { for } \theta_{i, i^{\prime}}+\max \left(0, g_{i, j}-g_{i^{\prime}, j^{\prime}}\right)<o_{i, j, i^{\prime}, j^{\prime}} \leqslant \\ & \theta_{i, i^{\prime}}+\min \left(g_{i, j}, c-g_{i^{\prime}, j^{\prime}}\right) \\ \max \left(0, g_{i, j}+g_{i^{\prime}, j^{\prime}}-c\right) & \text { for } \theta_{i, i^{\prime}}+\min \left(g_{i, j}, c-g_{i^{\prime}, j^{\prime}}\right)<o_{i, j, i^{\prime}, j^{\prime}} \leqslant \\ & \theta_{i, i^{\prime}}+\max \left(g_{i, j}, c-g_{i^{\prime}, j^{\prime}}\right) \\ g_{i^{\prime}, j^{\prime}}-c-\theta_{i, i^{\prime}}+o_{i, j, i^{\prime}, j^{\prime}} & \text { for } \theta_{i, i^{\prime}}+\max \left(g_{i, j}, c-g_{i^{\prime}, j^{\prime}}\right)<o_{i, j, i^{\prime}, j^{\prime}} \leqslant \\ & \theta_{i, i^{\prime}}+\min \left(0, g_{i, j}-g_{i^{\prime}, j^{\prime}}\right)+c .\end{cases}
$$

Finally,

$$
\begin{aligned}
& \operatorname{band}_{i^{\prime}, j^{\prime}, i, j}\left(g_{i, j}, g_{i^{\prime}, j^{\prime}}, c, \theta_{i, i^{\prime}}, o_{i, j, i^{\prime}, j^{\prime}}\right) \\
& =\operatorname{band}_{i, j, i^{\prime}, j^{\prime}}\left(g_{i, j}, g_{i^{\prime}, j^{\prime}}, c,-\theta_{i, i^{\prime}},-o_{i, j, i^{\prime}, j^{\prime}}\right) .
\end{aligned}
$$

Hence the bandwidth function for travel in the opposite direction, from intersection $i^{\prime}$ to intersection $i$, results from the original bandwidth function by applying the offset

$$
\widetilde{\Delta}:=-\Delta=-\mathrm{o}_{i, j, i^{\prime}, j^{\prime}}+\theta_{i, i^{\prime}}
$$

\subsection{Beyond pair-wise analysis}

Section 3.2 shows the bandwidth between adjacent intersections as a function of the intersection offsets. For an overall optimization, we require a model that seeks to maximise the bandwidth of all sections of road, which occur between adjacent intersections. The proposed preliminary model is as follows:

$$
\text { Maximise } Z_{0}=\sum_{\left(i, i^{\prime}, j, j^{\prime}\right)}\left(\beta_{i, i^{\prime}} \times \operatorname{band}_{i, j, i^{\prime}, j^{\prime}}+\beta_{i^{\prime}, i} \times \operatorname{band}_{i^{\prime}, j^{\prime}, i, j}\right)
$$

Subject to

$\operatorname{band}_{i, j, i^{\prime}, j^{\prime}}=\Psi\left(g_{i, j}, g_{i^{\prime}, j^{\prime}}, c, o_{i, j, i^{\prime}, j^{\prime}}, \theta_{i^{\prime}, i}\right)$ for all $i, i^{\prime}, j, j^{\prime} \mid i$ adjacent $i^{\prime}$, 
$\left|o_{i, j, i^{\prime}, j^{\prime}}-o_{i, j, i^{\prime}, j^{\prime}}^{\text {analytical }}\right| \leqslant \varepsilon \quad$ for all $i, i^{\prime}, j, j^{\prime} \mid i$ adjacent $i^{\prime}$,

$o_{i, j, i^{\prime}, j^{\prime}} \geqslant 0$

for all $i, i^{\prime}, j, j^{\prime} \mid i$ adjacent $i^{\prime}$.

Where

- band $_{i, j, i^{\prime}, j^{\prime}}$ is the bandwidth for travel between intersection $\boldsymbol{i}$ (phase $\mathfrak{j}$ ) and intersection $\boldsymbol{i}^{\prime}$ (phase $\boldsymbol{j}^{\prime}$ ),

- $\beta_{i, i^{\prime}}$ is the bandwidth priority, for travel between intersection $i$ and $i^{\prime}$, and

- $\Psi$ is a function to compute the bandwidth.

This model has a considerable number of decision variables. In some circumstances (e.g., for arterial roads) the number is reduced by replacing the comparative offset $o_{i, j, i^{\prime}, j^{\prime}}$ with an individual offset $o_{i}$ for each intersection. In this model constraint (17) has been added to ensure that the chosen offset lies within a given tolerance of the theoretical values $o_{i, j, i^{\prime}, j^{\prime}}^{\text {analytical }}$ which have been predetermined using alternative mathematical or empirical analysis. The model requires a function for the bandwidth (i.e., in terms of number of cars or time) and is ready for adaptation and additional technical constraints that may be imposed by the relevant traffic authority. The model's objective function is weighted bandwidth. The model attempts to balance the bandwidth over all sections of road, in each travel direction and for each phase. This approach is conceptually similar to a successful railway capacity analysis approach by Burdett and Kozan [5]. To see why this model is valid, consider the simplest scenario consisting of two adjacent sections of road and three intersections. Let these intersections be labelled $\boldsymbol{i}, \boldsymbol{i}+1$ and $\boldsymbol{i}+2$. To optimise the bandwidth for travel between intersection $i$ and $i+1$, suitable offsets must be chosen for intersection $i$ and $i+1$. Similarly to optimise the bandwidth for travel between intersection $i+1$ and $i+2$, suitable offsets must be chosen for intersection $i+1$ and $i+2$. In order to optimise the bandwidth for both sections of road, the offset at intersection $i+1$ is critical 
as it is associated with travel on two different sections of road. To choose this value without considering both sections of road will not result in an optimal solution. The proposed model handles the inherent complexities caused by these overlapping, and conceptually provides the best trade-off solution across the entire network (or arterial). Further implementation and testing is needed.

\section{A general optimisation and refinement approach}

In this section an alternative optimisation approach is proposed. This approach is different to the aforementioned bandwidth approach. It can be used to solve that problem, but the intention is solve more generic and comprehensive traffic control problems, that is, those that also involve cycle times and percentage splits, and any other decisions. This general approach is proposed to refine a current non-optimal starting solution and to find the optimal traffic signal timings. Our approach is shown in Algorithm 1.

\section{Algorithm 1 Optimisation strategy}

1. Construct a starting solution using an analytical model for "pair-wise intersections".

2. Evaluate starting solution using simulation

3. Begin optimisation routine

(a) Refine solution or population of solutions according to the selected optimisation technique

(b) Evaluate refinement(s) via separate simulations

(c) Compare candidate solutions and discard solutions that are "not promising"

(d) Repeat until convergence criteria or time limit is met.

4. Submit solution for verification and implementation. 
For the simulation we recommend the use of a fast approach that can be run within a fraction of a second or at most one to two seconds allowing multiple simulation runs. Multiple runs allows for the collection of more accurate statistics and quantifies the stochastic fluctuation between individual runs. Cellular automata was suggested during the Maths and Industry Study Group (MISG) as a good simulation approach. A review of the literature has since uncovered many alternative approaches of this sort [2, 20, 19, 21, 1, 9, 12, 15, for those published 2000-2010]. This optimisation approach is proposed because it can handle most "real-life" complexities. This is done via simulation. For example, stochastic travel times, different driving styles and habits, and road conditions. Other approaches are unable to model real life as accurately and hence are not able to accurately measure the "real" performance of specified signal timings. Simpler approaches are definitely useful and may provide useful solutions, but if the best solution is required, then the aforementioned approach will obtain it, provided that an appropriate simulator is used.

Numerous metrics can be used in this optimisation approach. However, the choice for practitioners is not trivial due to political and psychological aspects. We suggest the following metrics:

$$
\begin{aligned}
& \text { Minimise } Z_{1}=\sum_{r \in \Phi}\left(\text { delay }_{r}^{\text {avg }} \times \omega_{r}\right) ; \\
& \text { Minimise } Z_{2}=\sum_{r \in \Phi}\left(\text { pstop }_{r}^{\text {avg }} \times \omega_{r}\right) \text { where } \omega_{r}=T_{r}^{\text {min }} \\
& \text { Minimise } Z_{3}=\sum_{r \in \Phi}\left(\text { nstop }_{r}^{\text {avg }} / D_{r}\right) ; \text { and } \\
& \text { Minimise } Z_{4}=\sum_{r \in \Phi} \sum_{k}\left(\text { DistBetweenStops }_{r, k}^{\text {avg }} \times \omega_{r}\right)
\end{aligned}
$$

Where

- $\mathrm{k}$ is the index for vehicles $\mathrm{k}=1, \ldots, \mathrm{K}$.

- $l, l^{\prime}$ are the location indices. 
- $r$ is the index for routes.

- $\Phi$ is the set of routes. $\Phi=\left\{r=\left(l, l^{\prime}\right) \mid l, l^{\prime} \in \mathrm{L}\right\}$. The origin and destination locations points on each route are the input and output (IO) points of the road system.

- pstop $_{r, k}$ is the percentage number of stops for vehicle $k$ on route $r$.

- pstop $_{\mathrm{r}}^{\text {avg }}$ is the average percentage number of stops on route $r$.

- nstop $_{r, k}$ is the number of stops for vehicle $k$ travelling along route $r$.

- $\mathrm{D}_{\mathrm{r}}$ is the distance travelled on route $\mathrm{r}$.

- $\mathrm{T}_{\mathrm{r}}^{\min }$ is the minimum (unobstructed) time to travel on route $r$ that is, between IO points.

- $\omega_{r}$ is the priority weight for route $r$.

The four metrics are as follows:

- $Z_{1}$ minimises weighted average delays across the network, which is a measure for travel time;

- $Z_{2}, Z_{3}$ minimises weighted stops, which is a measure of driver dissatisfaction and fuel consumption;

- $\mathrm{Z}_{4}$ maximises the weighted distance between stops, which is a measure for "green flow" perception.

The purpose of the first criterion is to reduce the travelling time of all drivers in the network. This metric 'in theory' ensures that the delays experienced by drivers are minimal and throughput in the network is maximal. However, it has come to light via conversations with industry professionals (i.e., traffic signal engineers) that this metric is not always suitable. There are additional factors that need to be included that affect the quality of the signal timings and affect drivers perception of the systems efficiency. The first metric provides solutions that drivers often perceive as poor because it does not address the inconvenience of stopping at lights. The other metrics attempt to minimise 
this inconvenience and the resulting dissatisfaction. For example, drivers are perceived to be happier when there are fewer stops and the distance between stops is greater.

The following equations and constraints are necessary for the optimisation and to evaluate the aforementioned metrics,

$$
\begin{aligned}
& \mathrm{T}_{\mathrm{r}}^{\text {avg }}=\operatorname{eval}\left(\tilde{\mathbf{o}}, \tilde{\mathbf{c}}, \tilde{\mathrm{p}}_{1}, \tilde{\mathrm{p}}_{1}, \ldots, \tilde{\mathrm{p}}_{\mathrm{N}}\right) \quad \text { for all } \mathrm{r} \in \Phi, \\
& \text { delay }_{\mathrm{r}}^{\text {avg }}=\mathrm{T}_{\mathrm{r}}^{\text {avg }}-\mathrm{T}_{\mathrm{r}}^{\text {min }} \quad \text { for all } \mathrm{r} \in \Phi \text {, } \\
& \operatorname{nstop}_{\mathrm{r}, \mathrm{k}}=\operatorname{eval}\left(\tilde{\mathbf{o}}, \tilde{\mathbf{c}}, \tilde{\mathrm{p}}_{1}, \tilde{\mathrm{p}}_{1}, \ldots, \tilde{\mathrm{p}}_{\mathrm{N}}\right) \quad \text { for all } \mathrm{r} \in \Phi \text {, } \\
& \text { pstop }_{r, k}=\frac{\text { nstop }_{r, k}}{\text { nint }_{r}} \text { for all } k \in K_{r} \text {, for all } r \in \Phi \text {, } \\
& \text { pstop }_{\mathrm{r}}^{\text {avg }}=\frac{\sum_{\mathrm{k} \in \mathrm{K}_{\mathrm{r}}} \text { pstop }_{\mathrm{r}, \mathrm{k}}}{\mathrm{K}_{\mathrm{r}}} \text { for all } \mathrm{r} \in \Phi,
\end{aligned}
$$

DistBetweenStops $_{r, k}^{\text {avg }}=\frac{\sum_{z=1}^{\left|\lambda_{r, k}\right|} \operatorname{Dist}_{\left(u_{z}, u_{z+1}\right)}}{\left|\lambda_{r, k}\right|}$ for all $k \in K_{r}, r \in \Phi$,

$\left|o_{i, j, i^{\prime}, j^{\prime}}-o_{i, j, i^{\prime}, j^{\prime}}^{\text {analytical }}\right| \leqslant \varepsilon$ for all $i, i^{\prime}, j, j^{\prime} \mid i$ adjacent $i^{\prime}$, pstop $_{r}^{\text {avg }} \leqslant \tau_{r}$ or nstop avg $\leqslant \tau_{r}$ for all $r \in \Phi$,

$c_{i} \leqslant c_{i}^{\max } \quad i=1, \ldots, N \quad$ [Optional: solve for cycle time as well]

\section{Where}

- $\mathrm{N}$ is the number of intersections,

- $\boldsymbol{i}$ is the index for intersections, $\boldsymbol{i}=1, \ldots, \mathbf{N}$,

- $K_{r}$ is the number of vehicles on route $r$, which is equivalent to flow,

- $\mathrm{T}_{\mathrm{r}}^{\mathrm{avg}}$ is the average time to travel on route $\mathrm{r}$,

- $o_{i, j, i^{\prime}, j^{\prime}}^{\text {analytical }}$ is a "locally" optimal value of the offset, determined from the analytical approach, 
- $o_{i, j, i^{\prime}, j^{\prime}}$ is the offset between $(i, j)$ and $\left(i^{\prime}, j^{\prime}\right)$,

- $\varepsilon$ is the predefined tolerance for the offset (in seconds),

- nint $_{r}$ is the number of intersections along route $r$,

- $\tau_{r}$ is the tolerance for either the number or percentage number of stops on route $r$,

- $\lambda_{r, k}$ is the list of intersections where car $k$ stops on route $r, \lambda_{r, k}=$ $\left(u_{1}, u_{2}, \ldots\right)$,

- $\tilde{\mathrm{o}}$ is the list (vector) of offsets, $\tilde{\mathrm{o}}=\left(\mathrm{o}_{1}, \mathrm{o}_{2}, \ldots, \mathrm{o}_{\mathrm{N}}\right)$,

- $\tilde{\mathrm{c}}$ is the list (vector) of cycle times, $\tilde{\mathbf{c}}=\left(\mathrm{c}_{1}, \mathrm{c}_{2}, \ldots, \mathrm{c}_{\mathrm{N}}\right)$,

- $\tilde{p}_{i}$ is the percentage splits for intersection $i, \tilde{p}_{i}=\left(p_{i, 1}, p_{i, 2}, \ldots, p_{i, m_{i}}\right)$,

- $m_{i}$ is the number of phases at intersection $i$,

- Dist is the function to compute distance between two adjacent intersections, and

- EVAL represents the simulation procedure.

In some circumstances the priority (i.e., penalty) weighting is regarded as the flow, that is, $\omega_{\mathrm{r}} \equiv$ flow $_{\mathrm{r}}$. The offsets are restricted to be close to the analytical values because those values are optimal when the rest of the road network is ignored. Hence intuitively it would be appealing for those values to be selected if possible. However, what is meant by close is subjective. For some values of $\varepsilon$ it may be impossible to satisfy equation (28) across all intersections, and hence larger values of $\varepsilon$ should be trialled first. This tolerance is defined as a universal value but may also be allowed to differ for each intersection, that is, replace with $\varepsilon_{i}$ instead.

Some comments are now made about optimisation techniques. Many are available but Evolutionary and Simulated Annealing are well known, and are implemented relatively easily. They give good performance with standard control parameters. 
Optimisation problems with real value decision variables (like this one) have been efficiently solved using Differential Evolutionary Algorithms (DEA). The main idea behind DEA is the following perturbation operator that creates a new solution,

$$
\mathrm{o}_{\mathrm{i}}=\mathrm{a}_{\mathrm{i}}+\mathrm{F}\left(\mathrm{b}_{\mathrm{i}}-\mathrm{c}_{\mathrm{i}}\right) .
$$

Where $a, b$, and $c$ are three arbitrarily selected population members and $\mathrm{F}$ is a real number, typically between 0.1 and 1.2. This approach utilises "noise" and "diversity" within the population to create superior solutions. Superior solutions created by the aforementioned operator are kept while inferior solutions are rejected.

Simulated Annealing (SA) is an efficient optimisation approach as it operates upon (i.e., refines) a single solution. It is for this reason very "fast" computationally. The standard way to refine a real valued decision variable is,

$$
\mathrm{o}_{i}=\mathrm{a}_{\mathrm{i}} \pm \mathrm{r} \quad \text { where } \mathrm{r} \sim \mathrm{U}(0.1,5) .
$$

In both algorithms $o_{i}$ can be truncated to the nearest integer value when necessary. Previous SA solutions can also be recorded and then used as in the $\mathrm{EA}$, to more efficiently perturb the current solution. Random perturbations about the analytical value of the offsets should make a good starting point for either SA or DEA.

\section{Conclusions}

This article focusses on bandwidth quantification as part of a traffic control strategy. We sketched recent research trends, quantified the bandwidth for a pair-wise analysis of green phases at two intersections and outlined a general approach towards optimizing traffic signal times. Further testing is necessary to identify whether the proposed analytical method is operationally feasible, in particular for a reactive approach. The implementation of the proposed general optimisation framework is also necessary and it will require refinement 
and testing. Provided that a usable simulation tool is available, the time to perform these tasks can be reduced. Otherwise, further development time might be needed to implement a new simulation tool.

Acknowledgments We thank David Stewart of the Department of Transport and Main Roads for the chance to work on this problem at MISG. The following people worked on this project during MISG and we acknowledge their ideas and participation: Chen Chen, Lone Christoffersen, Jonathan Johansen, Phil Kilby, Erhan Kozan, Ruth Luscombe, Sam Nicol, Jimmi Phangestu, Claire Reeves, G'deona Soeharyo, David Shteinman, Phillip Stewart, Xuan $\mathrm{Vu}$.

Table 2: Bandwidth evaluation of the thirteen cases of Figure 2 quantified exactly. Note that $g_{i, j}=$ et - st and $g_{i^{\prime}, j^{\prime}}=$ et $^{\prime}-$ st $^{\prime}$ and $s t \equiv s t_{i, j}$, et $\equiv$ et $_{i, j}$, $\mathrm{st}^{\prime} \equiv \mathrm{st}_{\mathrm{i}^{\prime}, j^{\prime}}$, and et $^{\prime} \equiv \mathrm{et}_{\mathrm{i}^{\prime}, j^{\prime}}$. The full derivations of these equations are omitted for brevity.

\begin{tabular}{|c|c|c|c|}
\hline Case & Condition & Band & Equiv \\
\hline 1 & $\left(\mathrm{st}>\mathrm{st}^{\prime}\right) \wedge\left(\mathrm{et}^{\prime}<\mathrm{st}\right)$ & band $_{i, i^{\prime}}^{\text {time }}=0$ & na \\
\hline 2 & $\left(\mathrm{et}^{\prime}=\mathrm{st}\right) \wedge\left(\mathrm{st}^{\prime}<\mathrm{st}\right)$ & $\operatorname{band}_{i, i^{\prime}}^{\text {time }}=0$ & na \\
\hline 3 & $\left(\mathrm{st}^{\prime}<\mathrm{st}\right) \wedge\left(\mathrm{et}^{\prime}<\mathrm{et}\right):$ & & \\
\hline $3 a$ & $\left(0<\theta_{i, i^{\prime}} \leqslant \mathrm{et}^{\prime}-\mathrm{st}\right)$ & band $_{i, i^{\prime}}^{\text {time }}=$ et $^{\prime}-$ st $-\theta_{i, i^{\prime}}$ & Case B \\
\hline $\begin{array}{l}3 \mathrm{~b} \\
4\end{array}$ & $\begin{array}{l}\left(\theta_{i, i^{\prime}}>\mathrm{et}^{\prime}-\mathrm{st}\right) \\
\left(\mathrm{st}>\mathrm{st}^{\prime}\right) \wedge\left(\mathrm{et} \mathrm{t}^{\prime}=\mathrm{et}\right):\end{array}$ & $\operatorname{band}_{\mathfrak{i}, i^{\prime}}^{\text {time }}=0$ & na \\
\hline $4 a$ & $\left(0<\theta_{i, i^{\prime}} \leqslant g_{i, j}\right)$ & band $_{i, i^{\prime}}^{\text {time }}=$ et $^{\prime}-$ st $-\theta_{i, i^{\prime}}$ & Case B \\
\hline $\begin{array}{l}4 \mathrm{~b} \\
5\end{array}$ & $\begin{array}{l}\left(\theta_{i, i^{\prime}}>g_{i, j}\right) \\
\left(s t>s t^{\prime}\right) \wedge\left(e t<e^{\prime}\right):\end{array}$ & $\operatorname{band}_{i, i^{\prime}}^{\text {time }}=0$ & na \\
\hline $5 a$ & $\left(0<\theta_{i, i^{\prime}} \leqslant\right.$ et $^{\prime}-$ et $)$ & band $_{i, i^{\prime}}^{\text {time }}=$ et $^{\prime}-$ st & Case A \\
\hline $5 b$ & $\left(\mathrm{et}^{\prime}-\mathrm{et}<\theta_{i, i^{\prime}}<\mathrm{et}^{\prime}-\mathrm{st}\right)$ & $\operatorname{band}_{i, i^{\prime}}^{\text {time }}=$ et $^{\prime}-s t-\theta_{i, i^{\prime}}$ & Case B \\
\hline $\begin{array}{l}5 c \\
6\end{array}$ & $\begin{array}{l}\left(\theta_{i, i^{\prime}} \geqslant e t^{\prime}-s t\right) \\
\left(s t=s t^{\prime}\right) \wedge\left(e t^{\prime} \leqslant e t\right):\end{array}$ & $\operatorname{band}_{i, i^{\prime}}^{\text {time }}=0$ & na \\
\hline $\begin{array}{l}6 a \\
6 b\end{array}$ & $\begin{array}{l}\left(0<\theta_{i, i^{\prime}} \leqslant g_{i^{\prime}, j^{\prime}}\right) \\
\left(\theta_{i, i^{\prime}}>g_{i^{\prime}, j^{\prime}}\right)\end{array}$ & $\begin{aligned} \operatorname{band}_{i, i^{\prime}}^{\text {time }} & =\text { et }^{\prime}-\text { st }-\theta_{i, i^{\prime}} \\
\text { band }_{i, i^{\prime}}^{\text {time }} & =0\end{aligned}$ & $\begin{array}{l}\text { Case B } \\
\text { na }\end{array}$ \\
\hline
\end{tabular}




\begin{tabular}{|c|c|c|c|}
\hline Case & Condition & Band & Equiv \\
\hline 7 & $\left(\mathrm{st}^{\prime}=\mathrm{st}\right) \wedge\left(\mathrm{et}^{\prime}=\mathrm{et}\right):$ & & \\
\hline $7 a$ & $\left(0<\theta_{i, i^{\prime}} \leqslant g_{i, j}\right)$ & $\operatorname{band}_{i, i^{\prime}}^{\text {time }}=$ et $^{\prime}-\mathrm{st}-\theta_{i, i^{\prime}}$ & Case B \\
\hline $7 \mathrm{~b}$ & $\left(\theta_{i, i^{\prime}}>g_{i, j}\right)$ & band $_{i, i^{\prime}}^{\text {time }}=0$ & na \\
\hline 8 & $\left(\mathrm{st}=\mathrm{st}^{\prime}\right) \wedge\left(\mathrm{et}^{\prime}>\mathrm{et}\right):$ & & \\
\hline $8 a$ & $\left(0<\theta_{i, i^{\prime}}<g_{i, j}\right)$ & $\operatorname{band}_{i, i^{\prime}}^{\text {time }}=$ et $^{\prime}-\mathrm{st}-\theta_{i, i^{\prime}}$ & Case B \\
\hline $8 b$ & $\left(g_{i, j} \leqslant \theta_{i, i^{\prime}}<g_{i^{\prime}, j^{\prime}}\right)$ & band $_{i, i^{\prime}}^{\text {time }}=$ et $^{\prime}-\mathrm{st}-\theta_{i, i^{\prime}}$ & Case B \\
\hline $8 c$ & $\left(\theta_{i, i^{\prime}} \geqslant g_{i^{\prime}, j^{\prime}}\right)$ & $\operatorname{band}_{i, i^{\prime}}^{\text {time }}=0$ & na \\
\hline 9 & $\left(\mathrm{st}^{\prime}>\mathrm{st}\right) \wedge\left(\mathrm{et}^{\prime}<\mathrm{et}\right):$ & & \\
\hline $9 a$ & $\left(0<\theta_{i, i^{\prime}} \leqslant s t^{\prime}-s t\right)$ & $\operatorname{band}_{i, i^{\prime}}^{\text {time }}=$ et $^{\prime}-\mathrm{st} \equiv g_{i^{\prime}, j^{\prime}}$ & Case D \\
\hline $9 b$ & $\left(\mathrm{st}^{\prime}-\mathrm{st}<\theta_{\mathrm{i}, \mathrm{i}^{\prime}}<\mathrm{et}^{\prime}-\mathrm{st}\right)$ & band $_{i, i^{\prime}}^{\text {time }}=$ et $^{\prime}-\mathrm{st}-\theta_{i, i^{\prime}}$ & Case B \\
\hline $9 c$ & $\left(\theta_{i, i^{\prime}} \geqslant \mathrm{et}^{\prime}-\mathrm{st}\right)$ & $\operatorname{band}_{i, i^{\prime}}^{\text {time }}=0$ & na \\
\hline 10 & $\left(\mathrm{st}<\mathrm{st}^{\prime}\right) \wedge\left(\mathrm{et}^{\prime}=\mathrm{et}\right):$ & & \\
\hline $10 a$ & $\left(0<\theta_{\mathrm{i}, \mathrm{i}^{\prime}} \leqslant \mathrm{st}^{\prime}-\mathrm{st}\right)$ & $\operatorname{band}_{i, i^{\prime}}^{\text {time }}=\mathrm{et}^{\prime}-\mathrm{st} \equiv \mathrm{g}_{\mathrm{i}^{\prime}, j^{\prime}}$ & Case D \\
\hline $10 \mathrm{~b}$ & $\left(\mathrm{st}^{\prime}-\mathrm{st}<\theta_{i, i^{\prime}}<\mathrm{g}_{\mathrm{i}, \mathrm{j}}\right)$ & band $_{i, i^{\prime}}^{\text {time }}=$ et $^{\prime}-\mathrm{st}-\theta_{i, i^{\prime}}$ & Case B \\
\hline $10 c$ & $\left(\theta_{i, i^{\prime}} \geqslant g_{i, j}\right)$ & $\operatorname{band}_{i, i^{\prime}}^{\text {time }}=0$ & na \\
\hline
\end{tabular}

$11 \quad\left(\right.$ st $\left.<\mathrm{st}^{\prime}\right) \wedge\left(\right.$ et $\left.<\mathrm{et}^{\prime}\right):$

$11 \mathrm{a}\left(0<\theta_{i, i^{\prime}} \leqslant \mathrm{st}^{\prime}-\mathrm{st}\right) \wedge$ band $_{\mathrm{i}, \mathrm{i}^{\prime}}^{\mathrm{time}}=\mathrm{et}-\mathrm{st}^{\prime}+\theta_{\mathrm{i}, \mathrm{i}^{\prime}} \quad$ Case $\mathrm{C}$ $\left(\theta_{i, i^{\prime}} \leqslant\right.$ et $^{\prime}-$ et $)$

$11 \mathrm{~b} \quad\left(0<\theta_{i, i^{\prime}} \leqslant \mathrm{st}^{\prime}-\mathrm{st}\right) \quad \wedge \operatorname{band}_{\mathrm{i}, \mathrm{i}^{\prime}}^{\mathrm{time}}=\mathrm{et}^{\prime}-\mathrm{st}$

Case D

$\left(\theta_{i, i^{\prime}}>\right.$ et ${ }^{\prime}-$ et $)$

11c $\quad\left(\right.$ st $\left.^{\prime}-\mathrm{st}<\theta_{i, i^{\prime}}<\mathrm{et}^{\prime}-\mathrm{st}\right) \quad$ band $_{i, i^{\prime}}^{\text {time }}=\mathrm{et}^{\prime}-\mathrm{st}-\theta_{\mathrm{i}, \mathrm{i}^{\prime}}$

$11 d \quad\left(\theta_{i, i^{\prime}} \geqslant e^{\prime}-s t\right)$

$\operatorname{band}_{i, i^{\prime}}^{\text {time }}=0$

Case B

$12 \quad\left(\mathrm{st}<\mathrm{st}^{\prime}\right) \wedge\left(\right.$ et $\left.=\mathrm{st}^{\prime}\right)$ :

$12 a \quad\left(0<\theta_{i, i^{\prime}} \leqslant g_{i, j}\right)$

$\operatorname{band}_{i, i^{\prime}}^{\text {time }}=$ et - st $^{\prime}+\theta_{i, i^{\prime}}$

Case C

$12 \mathrm{~b} \quad\left(g_{i, j}<\theta_{i, i^{\prime}}<g_{i, j}+g_{i^{\prime}, j},\right)$

band $_{i, i^{\prime}}^{\text {time }}=$ et $^{\prime}-$ st $-\theta_{i, i^{\prime}}$

Case B

$12 \mathrm{c} \quad\left(\theta_{i, i^{\prime}} \geqslant g_{i, j}+g_{i^{\prime}, j^{\prime}}\right)$

$\operatorname{band}_{i, i^{\prime}}^{\text {time }}=0$

na

$13\left(\right.$ st $\left.<\mathrm{st}^{\prime}\right) \wedge\left(\right.$ et $\left.<\mathrm{st}^{\prime}\right)$ :

$13 \mathrm{a} \quad\left(\theta_{\mathrm{i}, \mathrm{i}^{\prime}} \leqslant \mathrm{st}^{\prime}-\mathrm{st}\right)$

$\wedge \operatorname{band}_{i, i^{\prime}}^{\text {time }}=0$

na

$\left(\theta_{i, i^{\prime}} \leqslant s t^{\prime}-\right.$ et $)$

$13 \mathrm{~b} \quad\left(\theta_{i, i^{\prime}} \leqslant s t^{\prime}-s t\right)$

$\wedge$ band $_{i, i^{\prime}}^{\text {time }}=$ et $^{\prime}-$ st $+\theta_{i, i^{\prime}} \quad$ Case C

$\left(\mathrm{st}^{\prime}-\mathrm{et}<\theta_{\mathrm{i}, \mathrm{i}^{\prime}} \leqslant \mathrm{et}^{\prime}-\mathrm{et}\right)$ 


\begin{tabular}{|c|c|c|c|}
\hline Case & Condition & Band & Equiv \\
\hline $13 c$ & $\begin{array}{l}\left(\theta_{i, i^{\prime}} \leqslant s t^{\prime}-s t\right) \\
\left(s t^{\prime}-\text { et } \theta_{i, i^{\prime}}\right) \\
\left(\theta_{i, i^{\prime}}>e^{\prime}-e t\right)\end{array}$ & band $_{i, i^{\prime}}^{\text {time }}=$ et $^{\prime}-\mathrm{st}^{\prime}$ & Case D \\
\hline $13 d$ & $\left(\mathrm{st}^{\prime}-\mathrm{st}<\theta_{\mathrm{i}, \mathrm{i}^{\prime}} \leqslant \mathrm{et}^{\prime}-\mathrm{st}\right)$ & band $_{i, i^{\prime}}^{\text {time }}=$ et $^{\prime}-\mathrm{st}-\theta_{i, i^{\prime}}$ & Case B \\
\hline $13 e$ & $\left(\theta_{i, i^{\prime}} \geqslant \mathrm{et}^{\prime}-\mathrm{st}\right)$ & $\operatorname{band}_{i, i^{\prime}}^{\text {time }}=0$ & na \\
\hline
\end{tabular}

\section{References}

[1] G. H. Bham and R. F. Benekohal. A high fidelity traffic simulation model based on cellular automata and car-following concepts.

Transportation Research Part C: Emerging Technologies, 12(1):1-32, 2004. doi:10.1016/j.trc.2002.05.001 M98

[2] E. Brockfeld, R. Barlovic, A. Schadschneider, and M. Schreckenberg. Optimizing traffic lights in a cellular automaton model for city traffic. Physical Review E-Statistical, Nonlinear, and Soft Matter Physics, 64(5 II):056132/1-056132/12, 2001. doi:10.1103/PhysRevE.64.056132 M98

[3] R. Burdett and E. Kozan. Evolutionary algorithms for flowshop sequencing with non-unique jobs. International Transactions in Operations Research, 7:401-418, 2000. doi:10.1111/j.1475-3995.2000.tb00207.x M86

[4] R. Burdett and E. Kozan. Evolutionary algorithms for resource constrained non-serial mixed flow shops. International Journal of Computational Intelligence and Application, 3(4):411-435, 2003. doi:10.1142/S1469026803001105 M86

[5] R. Burdett and E. Kozan. Techniques for absolute capacity determination in railways. Transportation Research B, 40(8):616-632, 2006. doi:10.1016/j.trb.2005.09.004 M96 
[6] B. De. Coensel, A. Can, B. Degraeuwe, I. De Vlieger, and D. Botteldooren. Effects of traffic signal coordination on noise and air pollutant emissions. Environmental Modelling and Software, 35:74-83, 2012. doi:10.1016/j.envsoft.2012.02.009 M82, M85

[7] A. Di Febbraro, D. Giglio, and N. Sacco. On applying petri nets to determine optimal offsets for coordinated traffic light timings. In IEEE 5th International Conference on Intelligent Transportation Systems, Singapore, 2002. doi:10.1109/ITSC.2002.1041317 M82, M83

[8] M. Gentili and P. B. Mirchandani. Locating sensors on traffic networks: models, challenges and research opportunities. Transportation Research C, 24:227-255, 2012. doi:10.1016/j.trc.2012.01.004 M82, M84

[9] R. Jiang and Q. S. Wu. A stopped time dependent randomization cellular automata model for traffic flow controlled by traffic light. Physica A: Statistical Mechanics and its Applications, 364:493-496, 2006. doi:10.1016/j.physa.2005.10.038 M98

[10] D. Johnston and D. Suter. The use of genetic algorithms to identify traffic conditions to optimise arterial performance. In 25th $A R R B$ Conference-Shaping the future: linking policy, research and outcomes. Perth, Australia., 2012. M79, M82, M84

[11] E. Kohler and M. Strehler. Combining static and dynamic models for traffic signal optimisation. In 15th meeting of the Euro Working Group on Transportation, Procedia Social and Behavioural Sciences, volume 54:1125-1134, 2012. doi:10.1016/j.sbspro.2012.09.827 M82, M85

[12] M. E. Larraga, J. A. Del Rio, and L. Alvarez-Lcaza. Cellular automata for one-lane traffic flow modeling. Transportation Research Part $C$ : Emerging Technologies, 13(1):63-74, 2005. doi:10.1016/j.trc.2004.12.001 M98

[13] P. Mirchandani and L. Head. A real time traffic signal control system: architecture, algorithms, and analysis. Transportation Research Part 


\section{C:415-432, 2001. doi:10.1016/S0968-090X(00)00047-4 M82, M83}

[14] T. Nagatani. Vehicular traffic through a sequence of green-wave lights. Physica A, 380:503-511, 2007. doi:10.1016/j.physa.2007.02.072 M82, M83

[15] T. Nagatani. Traffic states and fundamental diagram in cellular automaton model of vehicular traffic controlled by signals. Physica A: Statistical Mechanics and its Applications, 388(8):1673-1681, 2009. doi:10.1016/j.physa.2008.12.046 M98

[16] H. U. Peifeng, T. Zongzhong, Y. Zhenzhou, and J. Shunping. Variable-bandwidth progression optimization in traffic operation. Journal of Transportation Systems Engineering and Information Technology, 11(1):61-72, 2011. doi:10.1016/S1570-6672(10)60101-8 M79, M82, M84, M87

[17] J. Rohde and B. Friedrich. Offset optimizing with ctm and genetic algorithms: results from filed studies in Hannover. In 15th meeting of the Euro Working Group on Transportation, Procedia Social and Behavioural Sciences, volume 20:437-444, 2011. doi:10.1016/j.sbspro.2011.08.050 M82, M83

[18] J. J. Sanchez, M. J. Galan, and E. Rubio. Evolutionary computation applied to urban traffic optimisation. In Kosiski Witold, editor, Advances in Evolutionary Algorithms, page 468. I-Tech Education and Publishing, Vienna, Austria, November 2008. M80, M82, M83

[19] I. Spyropoulou. Modelling a signal controlled traffic stream using cellular automata. Transportation Research Part C: Emerging Technologies, 15(3):175-190, 2007. doi:10.1016/j.trc.2007.04.001 M98

[20] O. K. Tonguz, W. Viriyasitavat, and F. Bai. Modeling urban traffic: a cellular automata approach. Communications Magazine,IEEE, 47(5):142-150, 2009. doi:10.1109/MCOM.2009.4939290 M98 
[21] J. Wahle, L. Neubert, J. Esser, and M. Schreckenberg. A cellular automaton traffic flow model for online simulation of traffic. Parallel Computing, 27(5):719-735, 2001. doi:10.1016/S0167-8191(00)00085-5 M98

[22] W. M. Wey. Model formulation and solution algorithm of traffic signal control in an urban network. Computers, Environment and Urban Systems, 24:355-377, 2000. doi:10.1016/S0198-9715(00)00002-8 M81, M82

\section{Author addresses}

1. Robert Burdett, School of Mathematical Sciences, Queensland University of Technology, Australia.

mailto:r.burdett@qut. edu . au

2. Bradley Casey, School of Mathematical Sciences, Queensland University of Technology, Australia.

mailto:b.casey@qut.edu . au

3. Kai Helge Becker, School of Mathematical Sciences, Queensland University of Technology, Australia.

mailto:kai. becker@qut. edu . au 